\title{
Observation of electric-dipole-forbidden infrared transitions in cold molecular ions
}

\author{
Matthias Germann ${ }^{1}$, Xin Tong ${ }^{1,2}$ and Stefan Willitsch ${ }^{1 \star}$
}

\begin{abstract}
Spectroscopic transitions in atoms and molecules that are not allowed within the electric-dipole approximation, but occur because of higher-order terms in the interaction between matter and radiation, are termed dipole-forbidden ${ }^{1}$. These transitions are extremely weak and therefore exhibit very small natural linewidths. Dipole-forbidden optical transitions in atoms form the basis of next-generation atomic clocks ${ }^{2,3}$ and of high-fidelity qubits used in quantum information processors and quantum simulators ${ }^{4}$. In molecules, however, such transitions are much less characterized, reflecting the considerable challenges to address them. Here, we report direct observation of dipole-forbidden, electric-quadrupole-allowed infrared (IR) transitions in a molecular ion. Their detection was enabled by the very long interrogation times of several minutes afforded by the sympathetic cooling of individual quantum-state-selected molecular ions into the nearly perturbation-free environment of a Coulomb crystal. The present work paves the way for new mid-IR frequency standards and precision spectroscopic measurements on single molecules in the IR domain ${ }^{5}$.
\end{abstract}

Recent technological advances in the cooling and manipulation of molecules have opened up perspectives for new types of precision measurements. Fundamental questions, such as a possible time variation of fundamental physical constants ${ }^{6}$, the magnitude of the dipole moment of the electron ${ }^{7}$, the existence of additional fundamental interactions ${ }^{8}$ and the effects of parity-violating interactions in chiral molecules ${ }^{9}$, can now be addressed by molecular spectroscopy at an unprecedented precision.

Systems suited for precise spectroscopic measurements need to exhibit narrow spectral lines. Experiments need to allow for long interrogation times to minimize line broadening induced by the finite measurement time. Moreover, studies should be performed in a well-controlled and isolated environment. Trapped cold ions spatially localized in a Coulomb crystal ${ }^{10}$ with sufficiently strong confinement to allow Doppler-free excitation in the Lamb-Dicke regime fulfil these requirements. Together with ultracold atoms in optical lattices ${ }^{3}$, they represent one of the most advanced systems used in state-of-the-art precision spectroscopic measurements. Indeed, many of the currently most precise spectroscopic experiments rely on dipole-forbidden electronic transitions in Coulomb-crystallized atomic ions ${ }^{2,11}$. By contrast, to the best of our knowledge no dipole-forbidden vibrational-that is, IR-spectra of molecular ions have been reported so far. Studies of vibrational transitions in molecules, however, are attractive as they probe different spectral domains and dynamic regimes from those in studies of atomic systems $s^{5,8,12}$.

Dipole-forbidden vibrational transitions in molecules ${ }^{13}$ are several orders of magnitude weaker than dipole-forbidden optical transitions typically used in atoms $s^{2,3}$, rendering their observation challenging. Thus far, they were observed only in a handful of neutral diatomics, such as $\mathrm{H}_{2}, \mathrm{~N}_{2}$ and $\mathrm{O}_{2}$, using high-pressure samples and/or very long absorption path lengths ${ }^{14-16}$. These approaches, however, are not applicable to molecular ions because their charge and their high chemical reactivities set stringent limits on the achievable number densities.

More than six decades after their detection in neutral molecules ${ }^{14}$, we report the observation of dipole-forbidden singlephoton IR transitions in molecular ions using the prototypical homonuclear diatomic $\mathrm{N}_{2}^{+}$as an example. These molecules do not exhibit a dipole-allowed vibrational spectrum, because their dipole moment vanishes for all possible internuclear distances and therefore also does not change on vibration. In these systems, direct vibrational transitions are allowed within the electrical quadrupole approximation. The advantages offered by vibrational quadrupole transitions for precision measurements have recently been highlighted ${ }^{5,17}$ and, in particular, vibrational transitions in $\mathrm{N}_{2}{ }^{+}$have been suggested as attractive candidates for precision spectroscopic experiments ${ }^{18}$. Our approach capitalizes on the very long interrogation times (up to several minutes) enabled by the sympathetic cooling and subsequent spatial localization of individual $\mathrm{N}_{2}{ }^{+}$ions in a Coulomb crystal ${ }^{10}$. The sensitivity of the measurement is enhanced by confining the population of the molecules to a single rotational quantum state and using a highly sensitive charge-transfer $(\mathrm{CT})$ reaction scheme for the detection of absorption events ${ }^{19,20}$.

In our experiments, we focused on the observation of specific hyperfine components of the S(0) line of the IR fundamental excitation of $\mathrm{N}_{2}^{+}$-that is, the transition from the vibrational and rotational ground state $\left(v^{+\prime \prime}=0, N^{+\prime \prime}=0\right)$ to the second rotationally excited level $\left(N^{+\prime}=2\right)$ of the first vibrationally excited state $\left(v^{+\prime}=1\right)$ within the electronic ground state. Here, $v^{+}$and $N^{+}$denote the vibrational and rotational quantum numbers of the ion, and " and refer to the lower and upper level of the transition, respectively. The level scheme of $\mathrm{N}_{2}{ }^{+}$is depicted in Fig. 1. The $\mathrm{X}^{2} \Sigma_{\mathrm{g}}^{+}$electronic ground state of $\mathrm{N}_{2}{ }^{+}$may be well described within the Hund's case $b_{\beta_{\mathrm{J}}}$ angular momentum coupling scheme $\mathrm{e}^{21}$. The rotational angular momentum of the molecule $\mathbf{N}$ is first coupled with the total electron spin $\mathbf{S}$ to form $\mathbf{J}=\mathbf{N}+\mathbf{S}$. Here $S=1 / 2$, so that rotationally excited levels are split into two spin-rotation components. J couples to the nuclear spin $\mathbf{I}$ to form the total angular momentum $\mathbf{F}=\mathbf{J}+\mathbf{I}$. Even rotational levels of ${ }^{14} \mathrm{~N}_{2}{ }^{+}$are associated with the $I=0,2$ nuclearspin isomers (ortho- $\mathrm{N}_{2}{ }^{+}$). For the $I=2$ isomer, the levels are further split by the hyperfine interaction. The selection rules for the electric-quadrupole transitions are $\Delta F=0, \pm 1, \pm 2$ (ref. 22) with a propensity rule $\Delta N=\Delta J=\Delta F$ (ref. 21). In the case of $I=2$

${ }^{1}$ Department of Chemistry, University of Basel, Klingelbergstrasse 80, 4056 Basel, Switzerland, ${ }^{2}$ State Key Laboratory of Magnetic Resonance and Atomic and Molecular Physics, Wuhan Institute of Physics and Mathematics, Chinese Academy of Sciences, Wuhan 430071, China.

*e-mail: stefan.willitsch@unibas.ch 
a

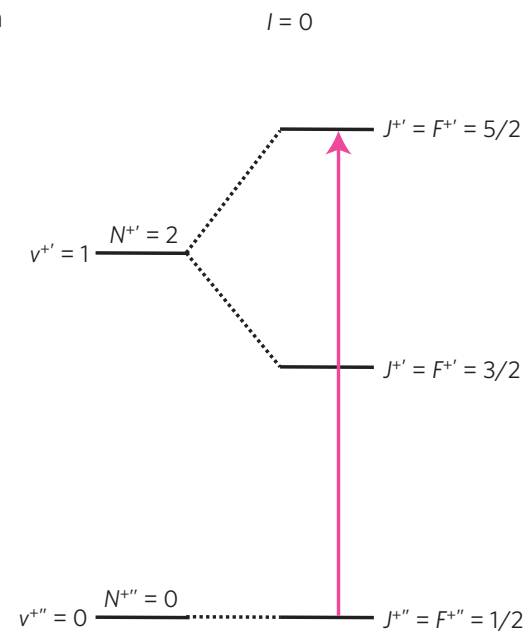

b

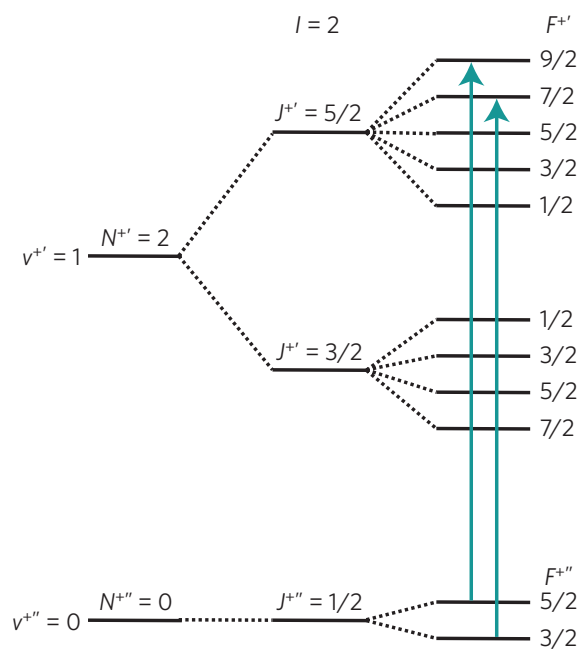

Figure 1 | Excitation scheme for $\mathbf{S}(0)$ electric-quadrupole rotational-vibrational transitions in $\mathbf{N}_{2}^{+}$. $\mathbf{a}, I=0$ nuclear-spin isomer. The excited level is split by the spin-rotation interaction. $\mathbf{b}, I=2$ nuclear-spin isomer. The levels are further split by the hyperfine interaction. The observed transitions (indicated by arrows) obey the propensity rule $\Delta F=\Delta J=\Delta N=2$. See text for explanation.

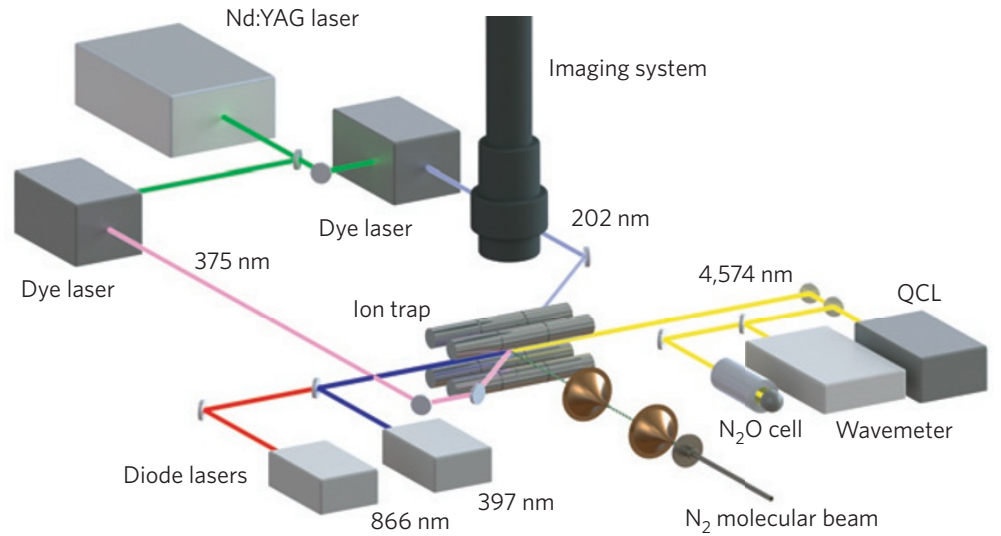

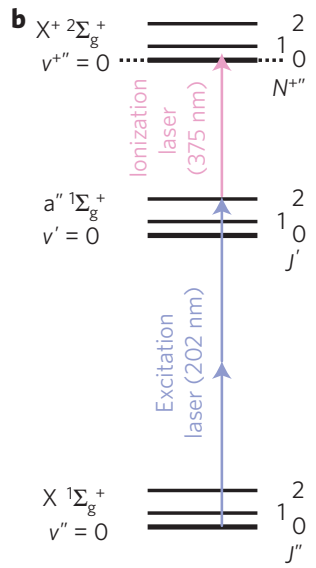

Figure 2 | Experimental set-up and state-selective photoionization scheme. a, Experimental set-up for the observation of dipole-forbidden vibrational transitions in $\mathrm{N}_{2}{ }^{+} . \mathrm{N}_{2}{ }^{+}$ions were loaded into a linear radiofrequency ion trap by state-selective photoionization of neutral $\mathrm{N}_{2}$ from a molecular beam and sympathetically cooled by simultaneously trapped laser-cooled $\mathrm{Ca}^{+}$ions to form bicomponent Coulomb crystals. The electric-quadrupole-allowed $\mathrm{S}(\mathrm{O})$ rotational-vibrational transition was excited with mid-IR radiation produced by a quantum-cascade laser (QCL). The fluorescence light of the $\mathrm{Ca}{ }^{+}$ions was collected by a CCD camera to image the crystals. $\mathbf{b}$, Resonance-enhanced [2+ $\left.1^{\prime}\right]$-photon threshold ionization scheme used to produce $\mathrm{N}_{2}{ }^{+}$ions state-selectively in the rotational-vibrational ground state $\left(v^{+\prime \prime}=0, N^{+\prime \prime}=0\right)$ : Neutral $\mathrm{N}_{2}$ molecules in the rovibronic ground state $\left(v^{\prime \prime}=0, J^{\prime \prime}=0\right)$ are excited in a two-photon transition to the $a^{\prime \prime}{ }^{1} \Sigma_{\mathrm{g}}^{+} v^{\prime}=0, J^{\prime}=2$ state. From this intermediate state, $\mathrm{N}_{2}$ is ionized by a third photon. State selectivity is ensured by setting the energy of the third photon to reach just slightly above the lowest ionization threshold (dotted line).

(statistical weight 5), one therefore expects two 'principal' transitions contributing to the $\mathrm{S}(0)$ line-that is, $F^{+\prime \prime}=3 / 2 \rightarrow F^{+\prime}=7 / 2$ and $F^{+\prime \prime}=5 / 2 \rightarrow F^{+\prime}=9 / 2$ (Fig. 1b). In the less abundant isomer $I=0$ (statistical weight 1 ), only the $J^{+\prime \prime}=F^{+\prime \prime}=1 / 2 \rightarrow J^{+\prime}=F^{+\prime}=5 / 2$ transition fulfils the propensity rule (Fig. 1a).

A schematic of the experimental set-up is presented in Fig. 2a. $\mathrm{N}_{2}{ }^{+}$ions were produced by photoionization of neutral $\mathrm{N}_{2}$ molecules from a molecular beam using a two-colour $\left[2+1^{\prime}\right]$ resonanceenhanced multiphoton ionization (REMPI) scheme (Fig. $2 b$ and Methods). By setting the energy of the third photon just above the lowest rovibrational ionization threshold of $\mathrm{N}_{2}$, it was ensured that the $\mathrm{N}_{2}{ }^{+}$ions were produced only in the $\mathrm{X}^{+2} \Sigma_{\mathrm{g}}^{+}, v^{+\prime \prime}=0, \mathrm{~N}^{+\prime \prime}=0$ rovibronic ground state ${ }^{19}$. Typically $20-25$ state-selected $\mathrm{N}_{2}{ }^{+}$ions were generated every experimental cycle and sympathetically cooled by the interaction with simultaneously trapped laser-cooled $\mathrm{Ca}^{+}$ ions. The cooled ions form bicomponent Coulomb crystals ${ }^{10}$ that were imaged by collecting the spatially resolved laser-cooling fluorescence of the $\mathrm{Ca}^{+}$ions (Fig. 3). Owing to the lighter mass of $\mathrm{N}_{2}{ }^{+}$compared to $\mathrm{Ca}^{+}$, the molecular ions formed a string along the trap axis, visible as a non-fluorescing core in the centre of the Coulomb crystal images (Fig. 3). The lifetime of the $N^{+\prime \prime}=0$ level of the state-selected $\mathrm{N}_{2}{ }^{+}$ions was determined to be $\approx 15$ min under our experimental conditions ${ }^{19}$, limited by collisions with residual background gas molecules ${ }^{20}$.

The $\mathrm{S}(0)$ electric-quadrupole transition in $\mathrm{N}_{2}{ }^{+}$was excited with mid-IR radiation produced by an external-cavity continuouswave quantum-cascade laser (QCL) operating at a wavelength of approximately $4.6 \mu \mathrm{m}$ (Methods). Vibrational excitation of the $\mathrm{N}_{2}{ }^{+}$ ions was detected by a CT reaction with Ar atoms according to $\mathrm{N}_{2}{ }^{+}\left(v^{+} \geq 1\right)+\mathrm{Ar} \rightarrow \mathrm{N}_{2}+\mathrm{Ar}^{+}$(ref. 19). For ions in the vibrational ground state, this reaction is endothermic and thus suppressed ${ }^{23}$. Therefore, the number of $\mathrm{N}_{2}{ }^{+}$ions removed from the dark crystal core by CT directly indicates the excitation fraction of $\mathrm{N}_{2}{ }^{+}$after IR irradiation at a certain wavelength. 


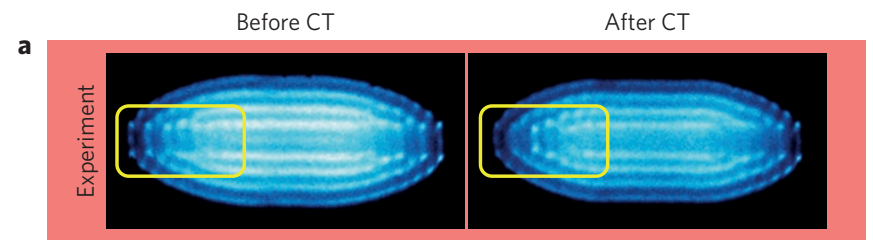

b

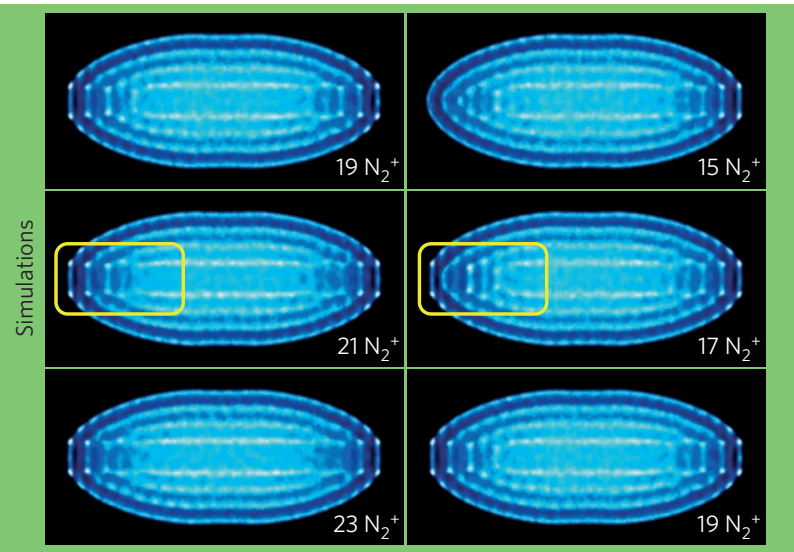

Figure 3 | Fluorescence images of bicomponent $\mathrm{Ca}+/ \mathrm{N}_{2}{ }^{+}$Coulomb crystals. a, Images taken during one experimental cycle before and after IR irradiation and CT detection of $\mathrm{N}_{2}{ }^{+}$ions. The $\mathrm{N}_{2}{ }^{+}$ions appear as a non-fluorescing core in the centre of the crystals. Slight changes in the structure of the core indicate the removal of $\mathrm{N}_{2}{ }^{+}$ions by CT reactions following their excitation (see highlighted regions). b, Synthetic images obtained from numerical simulations of the ion dynamics containing different numbers of $\mathrm{N}_{2}{ }^{+}$ions. The simulated images with 21 and $17 \mathrm{~N}_{2}{ }^{+}$ions best fit the experimental images taken before and after $\mathrm{CT}$, respectively.

To determine the number of $\mathrm{N}_{2}{ }^{+}$ions removed from the Coulomb crystals, the experimental images were compared against simulated images obtained from numerical simulations of the ion dynamics (Fig. 3). The comparison allowed the determination of the number of vibrationally excited $\mathrm{N}_{2}{ }^{+}$ions with an accuracy of \pm 1 (Fig. 3b). The spectrum in Fig. 4 was obtained by plotting the number of CT events observed after an irradiation time of $\approx 2 \mathrm{~min}$ as a function of the excitation frequency. Every data point represents an average of 8 or 16 independent measurements.

The spectrum shows two distinct features, a broader peak around $2,186.173 \mathrm{~cm}^{-1}[65,539.82 \mathrm{GHz}]$ and a narrower peak at $2,186.1804(4) \mathrm{cm}^{-1}[65,540.039(12) \mathrm{GHz}]$. The higher-frequency peak is assigned to the $F^{+\prime}=3 / 2 \rightarrow F^{+\prime}=7 / 2(I=2)$ transition on the basis of a comparison with its predicted position (indicated as solid vertical lines in Fig. 4). The predicted line positions were calculated from energy levels obtained by diagonalizing the hyperfine Hamiltonian from ref. 21 using the fine and hyperfine constants given in refs 21,24 as well as the vibrational and rotational constants obtained from high-resolution electronic spectra in ref. 25 (Methods). The shaded areas indicate the uncertainties of the theoretical line positions obtained from propagating the uncertainties in the spectroscopic constants. The width of this peak $(19 \mathrm{MHz}$ FWHM) is attributed to the bandwidth of the IR laser radiation (specified to be $<30 \mathrm{MHz}$ ). The Doppler width of approximately $1.3 \mathrm{MHz}$ at the secular ion temperature of $T_{\mathrm{sec}, \mathrm{N}_{2}+} \approx 20 \mathrm{mK}$ as well as the natural linewidth (see below) are much smaller. Collisional broadening is suppressed for trapped ions in ultrahigh vacuum. By contrast, the width of the lower-frequency peak (approximately $30 \mathrm{MHz}$ FWHM) is attributed to the partial overlap of the $F^{+\prime \prime}=5 / 2 \rightarrow F^{+\prime}=9 / 2(I=2)$ and the $J^{+\prime \prime}=1 / 2 \rightarrow J^{+\prime}=5 / 2(I=0)$ lines (Fig. 1), which are not resolved at the present laser bandwidth. The positions of these two transitions have been determined to be

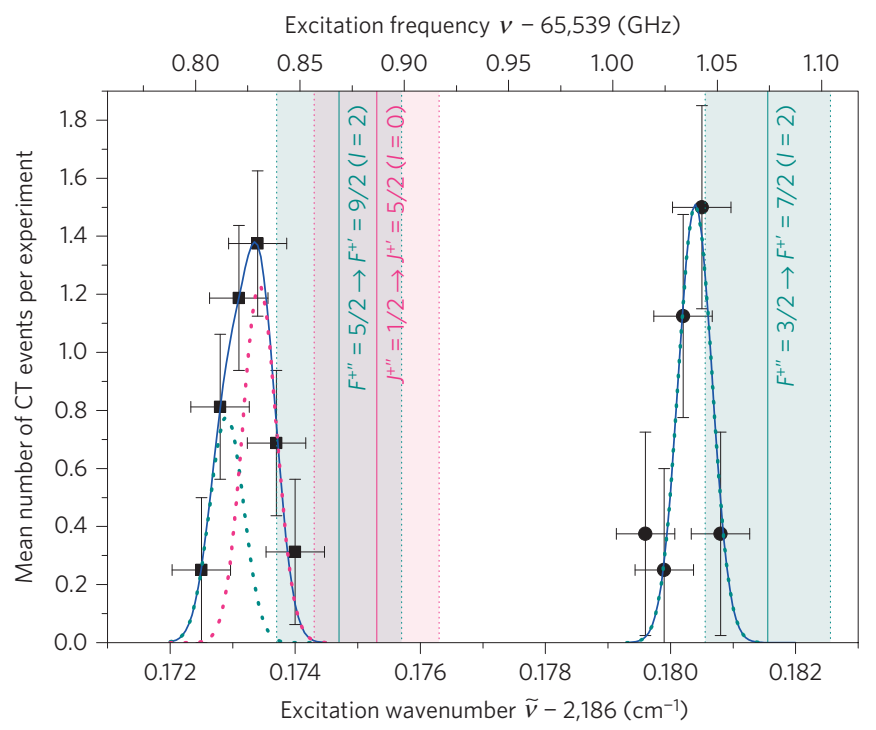

Figure 4 | Spectrum of hyperfine components of the $S(0)$ electric-quadrupole transition in ortho- $\mathrm{N}_{2}{ }^{+}$represented as the mean number of CT events per experimental cycle as a function of the excitation frequency. Data points marked with squares (circles) were averaged over 16 (8) experiments. The horizontal and vertical error bars correspond to the relevant $1 \sigma$ statistical uncertainties. The dotted lines represent Gaussian fits of the three spectral transitions to the data points and the solid blue line their sum. The solid green (red) vertical lines show predicted positions of the hyperfine transitions of the $I=2(I=0)$ isomer indicated in Fig. 1, based on spectroscopic constants taken from refs $21,24,25$. The shaded areas represent their $1 \sigma$ uncertainties. See text for details.

$2,186.1729(4) \mathrm{cm}^{-1}[65,539.815(12) \mathrm{GHz}]$ and $2,186.1734(4) \mathrm{cm}^{-1}$ $[65,539.831(12) \mathrm{GHz}]$, respectively, in a fitting procedure taking into account the theoretically expected line strengths of the different hyperfine components (Methods and Supplementary Information).

The predicted positions systematically appear at a slightly higher frequency than the observed ones. This may suggest that the presently observed vibrational frequency of $\mathrm{N}_{2}^{+}$and/or the rotational constant in the excited vibrational state are slightly lower than the value given in ref. 25 on which the prediction was based. Note that the vibrational frequencies of $\mathrm{N}_{2}^{+}$reported in the literature thus far were determined indirectly from electronic spectra and the values from recent studies vary by a few hundred $\mathrm{MHz}$ (refs 2426). The hyperfine splittings - that is, the relative positions of the three peaks-have been determined to be 16(1) MHz, 209(17) and 225(17) MHz. These findings are in reasonable agreement with the predictions of 18(1) MHz, 187(2) and 206(3) MHz, which are based on values of the hyperfine constants determined from radiofrequency (RF) and electronic spectroscopy in refs 21,24. Considering that the values of the rotational-vibrational and hyperfine constants reported in the literature vary significantly at the level of accuracy achieved in the present study, the agreement between the present observations and the predictions is satisfactory and sufficient to allow an assignment of the observed spectral features.

Based on the theory for the intensity of quadrupole vibrational transitions developed by Karl and $\mathrm{Poll}^{27}$ and the $\mathrm{N}_{2}{ }^{+}$quadrupole moments computed in ref. 28, we estimated the natural linewidth of the $v^{+\prime}=1, N^{+\prime}=2, J^{+\prime}=3 / 2, F^{+\prime}=7 / 2$ state to be $11 \mathrm{nHz}$, corresponding to a radiative lifetime of approximately $163 \mathrm{~d}$ (Supplementary Information). This translates into a vibrational excitation rate of $\approx 5$ ions s${ }^{-1}$ for 25 ions under the present experimental conditions. The observed excitation rate, however, is more than two orders of magnitude lower than the predicted rate. The reason for this discrepancy is unclear at present. It might lie 
in an overestimation of the IR laser beam intensity at the position of the ions, rotationally inelastic collisions with Ar atoms which may depopulate the initially prepared molecular state, inaccuracies of the theory, or a combination of these or other effects which may affect the observed CT rates. To conclusively ascertain whether experimental or theoretical factors are mainly responsible for this discrepancy, a high-level, systematic theoretical investigation of the quadrupole moment and quadrupole line strengths of $\mathrm{N}_{2}{ }^{+}$is highly desirable, but beyond the scope of the present study.

The present data represent the most precise measurement of vibrational transitions in $\mathrm{N}_{2}^{+}$yet. In combination with recently developed highly precise IR laser sources and frequency $\operatorname{combs}^{29}$, the present work opens up perspectives for novel IR precision spectroscopic measurements using molecular ions ${ }^{5,17,18}$. Furthermore, the capability to address such transitions paves the way for the implementation of molecular qubits which capitalize on the extremely long lifetime of the vibrational levels probed in the present study and take advantage of the superior coherence properties of magnetically insensitive molecular states, as recently discussed for the case of $\mathrm{HD}^{+}$in ref. 30. Another exciting perspective is the implementation of coherent, non-destructive schemes for detecting absorption events instead of the current charge-transfer method ${ }^{31}$. Thus, highly accurate spectroscopic measurements of extremely narrow spectroscopic transitions should become feasible in single molecules.

\section{Methods}

Experimental set-up. Our experimental set-up has been described in detail previously ${ }^{19,20}$. It consists of a linear RF ion trap operating at a RF amplitude $V_{\mathrm{RF}, 0-\mathrm{p}}=120 \mathrm{~V}$ and a RF frequency $\Omega_{\mathrm{RF}}=2 \pi \times 3.2 \mathrm{MHz}$. Static voltages $V_{\text {end }} \approx 1.5 \mathrm{~V}$ were applied for axial trapping. $\mathrm{N}_{2}{ }^{+}$ions were generated by photoionization of neutral $\mathrm{N}_{2}$ molecules from a molecular beam. $\mathrm{N}_{2}$ molecules in the rotational-vibrational ground state $\left(v^{\prime \prime}=0, J^{\prime \prime}=0\right)$ were first excited to the $a^{\prime \prime} \Sigma_{\mathrm{g}}^{+} v^{\prime}=0, J^{\prime}=2$ state using two photons at approximately $202 \mathrm{~nm}$. Subsequently, the $\mathrm{N}_{2}$ molecules were ionized by a third photon at approximately $375 \mathrm{~nm}$ to produce $\mathrm{N}_{2}{ }^{+}$ions in the absolute rotational-vibrational ground state ${ }^{19}$. The $\mathrm{N}_{2}{ }^{+}$ions were sympathetically cooled by the interaction with simultaneously trapped laser-cooled $\mathrm{Ca}^{+}$ions. The $\mathrm{Ca}^{+}$ions were loaded into the trap by photoionizing $\mathrm{Ca}$ atoms from an effusive Ca beam and were Doppler laser-cooled to a (secular) temperature of $T_{\mathrm{sec}, \mathrm{Ca} a^{+}} \approx 18 \mathrm{mK}$ using the $4 s^{2} \mathrm{~S}_{1 / 2} \rightarrow 4 p^{2} \mathrm{P}_{1 / 2}$ transition at $397 \mathrm{~nm}$. Population trapped in the $3 d^{2} \mathrm{D}_{3 / 2}$ state was repumped with an additional laser at $866 \mathrm{~nm}$. The fluorescence of the laser-cooled ions was collected with a microscope and detected by an electron multiplying charge-coupled device (EMCCD) camera to image the Coulomb crystals.

Excitation of quadrupole IR transitions. Vibrational transitions in $\mathrm{N}_{2}{ }^{+}$were excited with an external-cavity continuous-wave QCL (Daylight Solutions 21045-MHF) delivering a power of approximately $170 \mathrm{~mW}$ at a wavelength of $4,574 \mathrm{~nm}\left(\widehat{=} 2,186.2 \mathrm{~cm}^{-1}\right)$ with a nominal bandwidth $<0.001 \mathrm{~cm}^{-1}(<30 \mathrm{MHz})$. The mid-IR beam was focused to a spot size of approximately $140 \mu \mathrm{m}$ FWHM at the position of the $\mathrm{N}_{2}{ }^{+}$ions, resulting in an intensity of approximately $7.7 \times 10^{6} \mathrm{~W} \mathrm{~m}^{-2}$. The frequency of the QCL is monitored with a mid-IR wavelength meter (Bristol Instruments 621-A IR) with a quoted resolution of $8 \mathrm{MHz}$. The wavemeter is calibrated with an accuracy of $9 \mathrm{MHz}$ against absorption lines of $\mathrm{N}_{2} \mathrm{O}$ with frequencies known from the HITRAN database ${ }^{32}$. Stabilization of slow drifts in the QCL frequency is achieved with a proportional-integral servo loop based on the wavemeter measurements acting on the external-cavity grating at an update frequency of $\approx 1 \mathrm{~Hz}$.

Experimental procedure. A typical experiment started with the production of a pure $\mathrm{Ca}^{+}$Coulomb crystal of $\approx 1,000$ ions, followed by the loading of $20-25 \mathrm{~N}_{2}{ }^{+}$ ions. Afterwards, the mid-IR laser beam irradiated the $\mathrm{N}_{2}{ }^{+}$ions to excite the quadrupole transitions. Simultaneously, Ar gas was introduced into the chamber through a leak valve at a partial pressure of approximately $2 \times 10^{-8} \mathrm{mbar}$. After $2 \mathrm{~min}$, the leak valve was closed and the pressure in the chamber dropped to ultrahigh vacuum levels again. Images of the bicomponent Coulomb crystals were taken after loading the $\mathrm{N}_{2}^{+}$ions and after closing the leak valve (Fig. 3a). 'Blind' experiments, in which the QCL beam was blocked, were performed regularly in between the spectroscopic measurements to ascertain that no loss of $\mathrm{N}_{2}^{+}$ions other than CT occurred. In the very rare cases in which such losses were detected ( 2 out of 136 individual measurements that compose the data set shown in Fig. 4), a background loss rate was determined and subtracted from the actual CT rate. To locate the hyperfine components of the S(0) line, the QCL frequency was first scanned over adjacent intervals around the predicted line positions. On indication of CT, the interval was split up to further constrain the positions. Finally, the QCL frequency was fixed and the line profiles sampled in steps of $0.0003 \mathrm{~cm}^{-1}$. As a further check, we verified that no CT signal was observed when the QCL beam was present while being tuned off resonance.

Simulation of fluorescence images. To simulate experimental fluorescence images, classical trajectories of the ions for the present trapping and laser-cooling parameters were computed ${ }^{10}$. From the trajectories, three-dimensional histograms of the ion positions were generated, which were projected onto the image plane, taking into account the blurring of the images due to the finite focal depth of the microscope.

Fit of line positions. The positions of the three observed hyperfine transitions were determined in a global fit based on a quantitative theory for the hyperfine-resolved quadrupole line strengths (Supplementary Information). Three Gaussian functions were fitted to the observed transitions, with their relative line strengths constrained by the theoretical values. The linewidth, integrated intensity, line positions and hyperfine populations (which are not thermal, but initially determined by the hyperfine photoionization dynamics of the parent $\mathrm{N}_{2}$ molecules) were treated as free parameters in the fitting procedure.

Theoretical prediction of line positions. Following ref. 21, the Hamiltonian used to predict the hyperfine structure consists of spin-rotation, Fermi-contact hyperfine, dipolar hyperfine and quadrupolar hyperfine terms as well as a nuclear-spin-rotation term. Because of their small value $(<15 \mathrm{kHz})$ compared to the spectral resolution $(>10 \mathrm{MHz})$, the latter as well as the $\gamma_{N}$ and $t_{N}$ terms in the spin-rotation and dipolar hyperfine interactions, respectively, were neglected, as were matrix elements off-diagonal in the nuclear spin $I$ appearing in the quadrupolar hyperfine interaction. The Hamiltonian matrix was diagonalized within the Hilbert subspace of the $N^{+\prime}=0$ and $N^{+\prime}=2$ rotational levels for the ground and excited vibrational states, respectively, as mixing with adjacent rotational states was found to be negligible in ref. 21. Finally, to obtain the absolute level positions, the vibrational and rotational term energies were added using the molecular constants from ref. 25

\section{Received 14 January 2014; accepted 4 August 2014; published online 21 September 2014}

\section{References}

1. Bransden, B. H. \& Joachain, C. J. Physics of Atoms and Molecules 2nd edn (Prentice Hall, 2003).

2. Rosenband, T. et al. Frequency ratio of $\mathrm{Al}^{+}$and $\mathrm{Hg}^{+}$single-ion optical clocks; metrology at the 17th decimal place. Science 319, 1808-1812 (2008).

3. Hinkley, N. et al. An atomic clock with $10^{-18}$ instability. Science 341, 1215-1218 (2013).

4. Häffner, H., Roos, C. F. \& Blatt, R. Quantum computing with trapped ions. Phys. Rep. 469, 155-203 (2008).

5. Schiller, S., Bakalov, D. \& Korobov, V. I. Simplest molecules as candidates for precise optical clocks. Phys. Rev. Lett. 113, 023004 (2014).

6. Jansen, P., Bethlem, H. L. \& Ubachs, W. Tipping the scales: Search for drifting constants from molecular spectra. J. Chem. Phys. 140, 010901 (2014)

7. Baron, J. et al. Order of magnitude smaller limit on the electric dipole moment of the electron. Science 343, 269-272 (2014).

8. Salumbides, E. J. et al. Bounds on fifth forces from precision measurements on molecules. Phys. Rev. D 87, 112008 (2013).

9. Quack, M., Stohner, J. \& Willeke, M. High-resolution spectroscopic studies and theory of parity violation in chiral molecules. Annu. Rev. Phys. Chem. 59, 741-769 (2008).

10. Willitsch, S. Coulomb-crystallised molecular ions in traps: Methods, applications, prospects. Int. Rev. Phys. Chem. 31, 175-199 (2012).

11. Chou, C. W., Hume, D. B., Koelemeij, J. C. J., Wineland, D. J. \& Rosenband, T. Frequency comparison of two high-accuracy $\mathrm{Al}^{+}$optical clocks. Phys. Rev. Lett. 104, 070802 (2010).

12. Dickenson, G. D. et al. Fundamental vibration of molecular hydrogen. Phys. Rev. Lett. 110, 193601 (2013)

13. Papousek, D. Forbidden transitions in molecular vibrational-rotational spectroscopy. Collect. Czech. Chem. Commun. 54, 2555-2630 (1989).

14. Herzberg, G. Quadrupole rotation-vibration spectrum of the hydrogen molecule. Nature 163, 170 (1949).

15. Reid, J., Sinclair, R. L., Robinson, A. M. \& McKellar, A. R. W. Observation of electric quadrupole transitions in the fundamental band of $\mathrm{O}_{2}$ in the 1600-cm ${ }^{-1}$ region. Phys. Rev. A 24, 1944-1949 (1981).

16. Reuter, D., Jennings, D. E. \& Brault, J. W. The $v=1 \leftarrow 0$ quadrupole spectrum of $\mathrm{N}_{2}$. J. Mol. Spectrosc. 115, 294-304 (1986). 
17. Karr, J-Ph. $\mathrm{H}_{2}^{+}$and $\mathrm{HD}^{+}$: Candidates for a molecular clock. J. Mol. Spectrosc. 300, 37-43 (2014).

18. Kajita, M., Gopakumar, G., Abe, M., Hada, M. \& Keller, M. Test of $m_{p} / m_{e}$ changes using vibrational transitions in $\mathrm{N}_{2}^{+}$. Phys. Rev. A 89, 032509 (2014).

19. Tong, X., Winney, A. H. \& Willitsch, S. Sympathetic cooling of molecular ions in selected rotational and vibrational states produced by threshold photoionization. Phys. Rev. Lett. 105, 143001 (2010).

20. Tong, X., Wild, D. \& Willitsch, S. Collisional and radiative effects in the state-selective preparation of translationally cold molecular ions in ion traps. Phys. Rev. A 83, 023415 (2011).

21. Berrah Mansour, N. et al. Laser-rf double-resonance study of $\mathrm{N}_{2}^{+}$. Phys. Rev. A 44, 4418-4429 (1991)

22. Bunker, P. R. \& Jensen, P. Molecular Symmetry and Spectroscopy 2nd edn, 460 -462 (NRC Research Press, 2006).

23. Schlemmer, S., Kuhn, T., Lescop, E. \& Gerlich, D. Laser excited $\mathrm{N}_{2}^{+}$in a 22-pole ion trap: Experimental studies of rotational relaxation processes. Int. J. Mass Spectrom. 185, 589-602 (1999).

24. Scholl, T. J., Holt, R. A. \& Rosner, S. D. Fine and hyperfine structure in ${ }^{14} \mathrm{~N}_{2}^{+}$: The $\mathrm{B}^{2} \Sigma_{\mathrm{u}}^{+}-\mathrm{X}^{2} \Sigma_{\mathrm{g}}^{+}(0,0)$ band. J. Mol. Spectrosc. 192, 424-434 (1998).

25. Michaud, F., Roux, F., Davis, S. P., Nguyen, A-D. \& Laux, C. Q. High-resolution Fourier spectrometry of the ${ }^{14} \mathrm{~N}_{2}{ }^{+}$ion. J. Mol. Spectrosc. 203, 1-8 (2000).

26. Ferguson, D. W., Rao, K. N., Martin, P. A. \& Guelachvili, G. High resolution infrared Fourier transform emission spectra of the ${ }^{14} \mathrm{~N}_{2}^{+}$Meinel system: $\mathrm{A}^{2} \Pi_{\mathrm{u}}-\mathrm{X}^{2} \Sigma_{\mathrm{g}}^{+}$. J. Mol. Spectrosc. 153, 599-609 (1992).

27. Karl, G. \& Poll, J. D. On the quadrupole moment of the hydrogen molecule. J. Chem. Phys. 46, 2944-2950 (1967).

28. Bruna, P. J. \& Grein, F. The $\mathrm{X}^{2} \Sigma_{\mathrm{g}}^{+}$and $\mathrm{B}^{2} \Sigma_{\mathrm{u}}^{+}$states of $\mathrm{N}_{2}^{+}$: Hyperfine and nuclear quadrupole coupling constants, electric quadrupole moments, and electron-spin g-factors. A theoretical study. J. Mol. Spectrosc. 227, 67-80 (2004)
29. Hugi, A., Villares, G., Blaser, S., Liu, H. C. \& Faist, J. Mid-infrared frequency comb based on a quantum cascade laser. Nature 492, 229-233 (2012).

30. Bakalov, D., Korobov, V. \& Schiller, S. Precision spectroscopy of the molecular ion $\mathrm{HD}^{+}$: Control of Zeeman shifts. Phys. Rev. A 82, 055401 (2010)

31. Mur-Petit, J. et al. Temperature-independent quantum logic for molecular spectroscopy. Phys. Rev. A 85, 022308 (2012).

32. Rothman, L. et al. The HITRAN 2004 molecular spectroscopic database. J. Quant. Spectrosc. Radiat. Transfer 96, 139-204 (2005).

\section{Acknowledgements}

We would like to thank D. Wild, M. Grischa, P. Knöpfel, G. Holderied and A. Johnson for technical support. This work was supported by the University of Basel, the Swiss National Science Foundation as part of the National Centre of Competence in Research, Quantum Science \& Technology (NCCR-QSIT) and the European Commission under the Seventh Framework Programme FP7 GA 607491 COMIQ.

\section{Author contributions}

The experimental data were taken by X.T. and M.G. Synthetic images for data analysis were simulated by M.G. with advice from X.T. Data fitting and error analysis was done by M.G. as well as the calculation of theoretical line positions and intensities. S.W. conceived and supervised the project. All authors contributed to writing the manuscript.

\section{Additional information}

Supplementary information is available in the online version of the paper. Reprints and permissions information is available online at www.nature.com/reprints. Correspondence and requests for materials should be addressed to S.W.

\section{Competing financial interests}

The authors declare no competing financial interests. 